\title{
Respostas anatômicas de Eumachia kappleri em três áreas da Amazônia Mato-Grossense
}

\begin{abstract}
Eumachia DC. é um gênero de Rubiaceae que recentemente teve sua circunscrição ampliada devido a estudos baseados em dados moleculares e distribuição geográfica. Rubiaceae, representanda por 13.235 espécies distribuídas em 614 gêneros é uma das maiores famílias de Angiospermas. Diante dessa grande diversidade e presença em diferentes estratos vegetacionais, espécies da família Rubiaceae possuem grande importância para estudos relacionados a ecologia, conservação e preservação em regiões tropicais. A anatomia ecológica pode ser uma abordagem útil para compreender as mudanças estruturais e entender os processos de colonização e sobrevivência de espécies. O objetivo deste estudo foi comparar a anatomia foliar de Eumachia kappleri (Miq.) Delprete \& J.H. Kirkbr., ocorrente em três diferentes áreas, buscando identificar caracteres-chave que mostrem estratégias adaptativas em seus ambientes naturais. Os indivíduos foram coletados em três áreas inseridas em uma região onde ocorrem duas estações climáticas definidas. Para o estudo dos caracteres anatômicos, em cada área naturais. Os indivíduos foram coletados em três áreas inseridas em uma região onde ocorrem duas estações climáticas definidas. Para o estudo dos caracteres anatômicos, em cada área
foram identificados três indivíduos e, destes, coletadas cinco folhas adultas, antes totalmente expostas ao sol, que foram fixadas em FAA50 por 48 horas e depois conservadas em etanol $70 \%$. Posteriormente, com técnicas usuais em anatomia vegetal e com uso de lâminas de aço, foram obtidas secções transversais e procedeu-se a dissociação de epidermes. Lâminas histológicas semi-permanentes foram montadas, para visualização e registro fotográfico em fotomicroscópio fotônico trinocular acoplado a um computador. Para o estudo quantitativo serviram onze atributos anatômicos, analisados com o auxílio do software de análise de imagem. Após normalizar os dados necessários, uma matriz de correlação foi construída e posteriormente uma MANOVA foi realizada. Após identificar quais variáveis colaboraram para a significância da MANOVA, foi aplicado o teste de Tukey. Através da MANOVA foi possíve identificar diferença significativa entre as áreas. Na maioria das variáveis morfoanatômicas os indivíduos da área Reserva FA (Reserva do Floresta Amazônica Hotel Resort) apresentaram medidas dificín

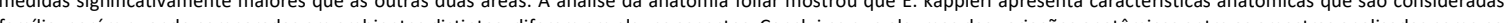

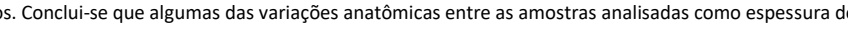
epiderme e mesofilo favorecem a aclimatação da espécie às clareiras florestais, tornando-a bem ajustada em ambientes abertos.
\end{abstract}

\section{Anatomical responses of Eumachia kappleri in three areas of Mato Grosso Amazon}

\begin{abstract}
Eumachia DC. is a genus from Rubiaceae that, recently, had its circumscription enlarged due to studies based on molecular data and geographic distribution. Rubiaceae is one of the largest families of Angiosperms, and it is represented by thirteen thousand two hundred and thirty-five species, distributed in six hundred and fourteen genera. Because of their great diversity and presence in different vegetative strata, species of the Rubiaceae family are of great importance for studies related to ecology, conservation and preservation in tropical regions. Ecological anatomy may be a useful approach to the understanding of the structural changes and the species colonization and survival processes. The aim of this study was to compare the foliar anatomy of the Eumachia kappleri (Miq.) Delprete \& J.H. Kirkbr., which occurrs in three different areas, seeking to identify key characters that might present adaptive strategies in their natural environments. The individuals environments. out. For visualization and photographic recording, semi-permanent histological slides were assembled, and a trinocular photomicroscope coupled to a computer was used for that. For the quantitative study, eleven anatomical attributes were considered and analyzed with the aid of an image analysis software. After normalizing the required data, a correlation matrix was constructed and, subsequently, a MANOVA was performed. After identifying the variables that contributed to the significance of the MANOVA, the Tukey test was applied. Through the MANOVA it was possible to identify a significant difference between the areas. In most of the morpho-anatomical variables, the individuals from the reserve area FA (Amazon Forest Reserve Hotel Resort) presented significantly larger measures than the other from the two areas. The analysis of the foliar anatomy showed that E. kappleri presents anatomical characteristics that are considered common to the family, however, when compared in different environments, they differ in some aspects. It is concluded that some of the anatomical variations between the analyzed samples, such as the thickness of the epidermis and mesophyll, favor the acclimatization of the species to the forest clearings, making it well-adjusted in open environments.
\end{abstract}

Keywords: Adaptation; Eumachia; Anatomical characters; Plasticity.

Topic: Conservação da Biodiversidade

Reviewed anonymously in the process of blind peer

Virgilia Aparecida Pegorini Rocha

Universidade do Estado de Mato Grosso, Brasil

http://lattes.cnpq.br/1105523955066811

virgilia pegorini@hotmail.com

Andréia Anjo Pereira

Universidade do Estado de Mato Grosso, Brasil

http://lattes.cnpq.br/3250978697350446

andreia_biol@hotmail.com

Karla Bianca de Deus Bento (iD)

Universidade do Estado de Mato Grosso, Brasil

http://lattes.cnpq.br/2740048991857611

http://orcid.org/0000-0002-2208-911X

karllakiva1@ @hotmail.com

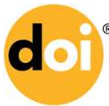

DOI: 10.6008/CBPC2179-6858.2019.002.0008
Received: 12/02/2019

Approved: 28/03/2019
Odair de Souza Fagundes

Universidade do Estado de Mato Grosso, Brasil

http://lattes.cnpq.br/4560356371813646

odair.bio.afmt@gmail.com

Norberto Gomes Ribeiro Junior (ic

Universidade do Estado de Mato Grosso, Brasil

http://lattes.cnpq.br/0708897518142620

http://orcid.org/0000-0002-7518-7952

norberto.gomes@unemat.br

Ivone Vieira da Silva

Universidade do Estado de Mato Grosso, Brasil

http://lattes.cnpq.br/4828497736925374

ivibot@hotmail.com

Referencing this:

ROCHA, V. A. P.; PEREIRA, A. A.; BENTO, K. B. D.; FAGUNDES, O. S.; RIBEIRO JUNIOR, N. G.; SILVA, I. V.. Respostas anatômicas de Eumachia kappleri em três áreas da Amazônia Mato-Grossense. Revista Ibero Americana de Ciências Ambientais, v.10, n.2, p.74-82, 2019. DOI: http://doi.org/10.6008/CBPC2179-6858.2019.002.0008 


\section{INTRODUÇÃO}

Eumachia DC. é um gênero da família Rubiaceae composto por 83 espécies com distribuição pantropical que recentemente teve sua circunscrição ampliada devido a estudos baseados em dados moleculares (ANDERSSON et al., 1999; NEPOKROEFF et al., 1999; ANDERSSON, 2001), portanto, atualmente o gênero consiste principalmente de espécies que foram removidas dos gêneros Margaritopsis C. Wright e Psychotria L. (ANDERSSON, 2001). Eumachia é formado por espécies arbustivas de aproximadamente $1 \mathrm{~m}$ de altura, que ocorrem comumente no sub-bosque de Florestas Ombrófilas.

As folhas de Rubiaceae, são caracterizadas anatomicamente por mesofilo dorsiventral, são hipostomáticas com estômatos paracíticos, organização do sistema vascular do pecíolo e da nervura principal em arco e presença de feixes acessórios (METCALFE et al., 1979; MORAES et al., 2011; ZINI et al., 2016). Porém, sabe-se que variações na estrutura das plantas como resposta funcional às pressões ambientais são particularmente mais expressivas na morfologia e anatomia foliar (e.g. aumento da intensidade luminosa, aumento da densidade estomática) (CAO, 2000; CABRAL et al., 2018).

Espécies da família como as dos gêneros Genipa L. e Psychotria L., podem ter grande capacidade de apresentar diferenças fenotípicas e alta capacidade de adaptação a ambientes de sol e sombra, o que pode proporcionar vantagem competitiva em ecossistemas dinâmicos e perturbados (CAMPBELL et al., 2018). Essa plasticidade morfológica implica uma capacidade eficiente para da planta a aquisição de recursos, já a maior plasticidade fisiológica está relacionada a uma maior capacidade de explorar ambientes de alta luminosidade (RABELO et al., 2013).

A anatomia ecológica pode ser uma abordagem útil para compreender as mudanças estruturais e entender os processos de colonização e sobrevivência de espécies, apontando adaptações relacionadas ao ambiente, pois o conhecimento da estrutura interna da planta indica e explica sua adaptação a determinado ambiente (ZINI et al., 2016; GAVILANES et al., 2016; SILVA et al., 2017; DARDENGO et al., 2017).

Neste contexto, este estudo teve como objetivo comparar a anatomia foliar de Eumachia kappleri (Miq.) Delprete \& J.H. Kirkbr., uma espécie de sub-bosque, ocorrente em três diferentes áreas da Amazônia mato-grossense, buscando identificar caracteres-chave que mostrem estratégias adaptativas em seus ambientes naturais. Apresenta-se a hipótese de que ocorra influência do espaço ou de fatores ambientais sobre as características anatômicas das populações, com diferença entre indivíduos de áreas distintas.

\section{MATERIAIS E MÉTODOS}

\section{Áreas estudadas}

As áreas de estudo estão inseridas em uma região onde ocorrem duas estações climáticas definidas, uma chuvosa de outubro a abril e uma de estiagem de maio a setembro, com precipitação média anual de $2.232 \mathrm{~mm}$ e temperatura média anual de $26-27^{\circ} \mathrm{C}$ (DUBREUIL et al., 2012). Os indivíduos coletados foram encontrados próximos à clareiras ou aberturas de trilhas nas três áreas (Fig. 1). 

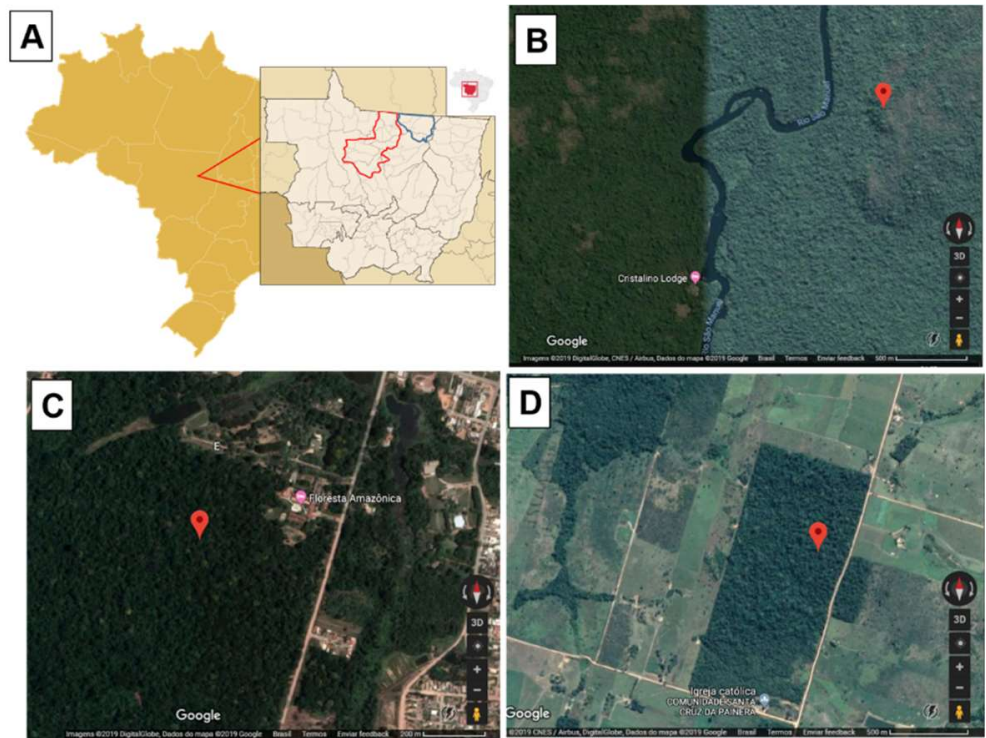

Figura 1: Áreas de coleta. A- Estado de Mato Grosso, município de Alta Floresta em vermelho; município de Novo Mundo em Azul. B- Reserva do Floresta Amazônica Hotel Resort - Reserva FA; C- Parque C-E; D- Reserva Particular do Patrimônio Natural - RPPN Mirante da Serra.

A primeira área, denominada Reserva do Floresta Amazônica Hotel Resort (Reserva FA), está situada no perímetro urbano do município de Alta Floresta- Mato Grosso, sob as coordenadas $9^{\circ} 52^{\prime} 44.6^{\prime \prime S}$ e 5605'57.3"W. A área abrange cerca de 50 hectares de Floresta Ombrófila Densa (EMBRAPA, 2018) com perturbação antrópica média (Fig. 1 B). Os indivíduos coletados estão a aproximadamente 500 metros de distância do curso d'água existente no local.

A segunda área de estudo, um parque municipal também localizado no perímetro urbano da cidade de Alta Floresta, denominado Parque C-E (S 09 52' 38.07” W 05605' 29.75”, possui 13 hectares de Floresta Ombrófila Densa (EMBRAPA, 2018), com alto grau de perturbação (Fig. 1C). Nessa área os indivíduos foram coletados a aproximadamente 50 metros do curso d'água.

Já a terceira área é uma Reserva Particular do Patrimônio Natural (RPPN) Mirante da Serra, localizada no município de Novo Mundo e sob as coordenadas 09³5'11"S, 5555'10"W (Fig. 1D). Essa área juntamente com outras três unidades de preservação, totalizam cerca de 6.500 hectares de vegetação com pouca/nenhuma perturbação antrópica (RPPN's Cristalino) (FUNDAÇÃO CRISTALINO, 2018). A coleta foi realizada a cerca de 300 metros de distância do rio Cristalino, em um ambiente úmido, com pouca influência visível de seca e sem inundações na estação chuvosa (SASAKI et al., 2010). A vegetação predominante é de Floresta Ombrófila Densa Submontana e Floresta Estacional Semidecidual (IBGE, 2012; SEMA, 2010).

\section{Coleta de dados}

Para o estudo dos caracteres anatômicos de Eumachia kappleri as coletas foram realizadas entre março e abril de 2018. Em cada área, três indivíduos foram identificados, e destes, retiradas cinco folhas adultas que estavam totalmente expostas ao sol. As folhas foram fixadas em FAA 50 por 48 horas e depois conservadas em etanol 70\% (JOHANSEN, 1940). Além das amostras utilizadas para estudo anatômico, outras amostras formaram material que foi depositado no Herbário da Amazônia Meridional (HERBAM), sob o voucher HERBAM 20592. 


\section{Anatomia Foliar}

Para análise anatômica se prestaram técnicas usuais em anatomia vegetal e cortes a mão livre, com uso de lâminas de aço. Assim, foram obtidas secções transversais da região mediana da folha. As secções passaram por clarificação em hipoclorito de sódio a $2 \%$ e posteriormente por tripla lavagem com água destilada. Em seguida, coradas com azul de Astra e fucsina básica (KRAUS et al., 1997). Lâminas histológicas semi-permanentes foram montadas, para visualização e registro fotográfico em fotomicroscópio fotônico trinocular Leica ${ }^{\circledR}$ ICC50, acoplado a um computador e com auxílio do software LAZ EZ 1.7.0.

A dissociação epidérmica se deu através do método de Jeffrey modificado (KRAUS et al., 1997), onde porções foliares de aproximadamente $1 \mathrm{~cm}^{2}$ foram inseridas em microtubos com solução formada por peróxido de hidrogênio (Volume 30) e ácido acético glacial na proporção de 1:1, mantidas em estufa a 65C por aproximadamente 12 horas, para isolamento das epidermes. Após esse período, as amostras passaram por lavagem em água destilada e, com o auxílio de um pincel, separadas as duas superfícies epidérmicas que foram coradas com safranina para montagem das lâminas histológicas. Os atributos anatômicos das secções transversais e paradérmicas foram medidos com auxílio do software de análise de imagem Anati Quanti (AGUIAR et al., 2007).

\section{Análise de Dados}

Inicialmente testaram-se os pressupostos de normalidade e homocedasticidade usando o software PAST (Paleontological Statistics), versão 3.15 (HAMMER et al., 2001). A partir da transformação dos valores dos atributos anatômicas antes não-normais (Tabela 1), utilizou-se o software R v. 3.4.3 (R CORE TEAM, 2017) para os testes estatísticos. Uma matriz de correlação foi construída para testar as correlações entre as variáveis dependentes usando a função $\operatorname{cor}()$ do pacote stats (R CORE TEAM, 2017), sendo consideradas correlacionadas variáveis com valor de correlação acima de $|0,70|$ e eliminada uma delas para testes posteriores; para eliminação usou-se como critério a variável com maior sentido biológico para as hipóteses testadas.

Tabela 1: Atributos anatômicos foliares, suas definições e transformações necessárias para teste estatístico.

\begin{tabular}{|l|l|l|}
\hline Variável anatômica & Definição & Transformação de dados \\
\hline EAD $(\mu \mathrm{m})$ & Espessura de epiderme adaxial & ----- \\
\hline EAB $(\mu \mathrm{m})$ & Espessura de epiderme abaxial & Log 10 \\
\hline EPa $(\mu \mathrm{m})$ & Espessura do parênquima paliçádico & Box-cox \\
\hline Ela $(\mu \mathrm{m})$ & Espessura do parênquima lacunoso & Box-cox \\
\hline EMe $(\mu \mathrm{m})$ & Espessura do mesofilo & Log 10 \\
\hline EFNC $(\mu \mathrm{m})$ & Espessura do feixe vascular da nervura central & Vy \\
\hline Dest $\left(\right.$ estômatos $\left.\mu \mathrm{m}^{-2}\right)$ & Densidade estomática & Log 10 \\
\hline $\mathrm{IE}$ & Índice estomático & ----- \\
\hline $\mathrm{MEE}(\mu \mathrm{m})$ & Medida equatorial do estômato & ----- \\
\hline $\mathrm{MPE}(\mu \mathrm{m})$ & Medida polar do estômato & ----- \\
\hline $\mathrm{AO}(\mu \mathrm{m})$ & Abertura do ostíolo & ----- \\
\hline
\end{tabular}

Seguidamente, testou-se o valor de inflação das variáveis (VIF) através da função vif() do pacote car (FOX et al., 2011) e constatamos que não haviam variáveis inflando o modelo (VIF < 10). Uma MANOVA foi realizada (Multivariate Analysis of Variance) com os atributos anatômicos remanescentes para detectar 
possível diferença entre as três áreas utilizando a função manova (cbind()) do pacote $d p l y r$ (WICKHAM et al., 2018). Após identificar quais variáveis colaboraram para a significância da MANOVA com a função summary.aov() realizamos um teste post-hoc de Tukey através da função TukeyHSD() do pacote stats (R CORE TEAM, 2017).

Para todos os atributos anatômicos calculamos médias e desvio-padrão usando a função summaryBy() do pacote doBy (HøJSGAARD et al., 2010). De posse dos dados morfoanatômicos foliares, organizamos uma tabela com a média das variáveis acompanhadas do desvio padrão de Eumachia kappleri provenientes das três áreas de vegetação nativa.

\section{RESULTADOS}

Mediante a matriz de correlação, foi possível identificar dois atributos a serem descartados (Tabela 2).

Tabela 2: Matriz de correlação de Pearson entre os atributos anatômico de amostras de Eumachia kappleri provenientes de três áreas de vegetação natural na Amazônia mato-grossense. Definição das siglas na tabela 1.

\begin{tabular}{|c|c|c|c|c|c|c|c|c|c|c|c|}
\hline & Ead & Eab & Epa & Ela & Eme & EFNC & Dest & IE & MEE & MPE & $\mathrm{AO}$ \\
\hline Ead & & 0,41 & 0,64 & 0,5 & 0,57 & 0,31 & $-0,14$ & 0,04 & $-0,12$ & 0,05 & 0,15 \\
\hline Eab & & & 0,47 & 0,47 & 0,53 & 0,38 & $-0,1$ & 0,14 & $-0,23$ & $-0,01$ & 0,1 \\
\hline Epa & & & & 0,51 & 0,66 & 0,34 & $-0,17$ & 0,07 & $-0,12$ & 0,19 & $-0,03$ \\
\hline Ela & & & & & 0,95 & 0,44 & $-0,13$ & 0,1 & $-0,04$ & 0,11 & 0,23 \\
\hline Eme & & & & & & 0,49 & $-0,15$ & 0,11 & $-0,04$ & 0,14 & 0,21 \\
\hline EFNC & & & & & & & $-0,15$ & 0,11 & $-0,05$ & 0,14 & $-0,09$ \\
\hline Dest & & & & & & & & 0,79 & 0,06 & $-0,06$ & 0,01 \\
\hline IE & & & & & & & & & 0,12 & 0,2 & 0,09 \\
\hline MEE & & & & & & & & & & 0,6 & 0,36 \\
\hline MPE & & & & & & & & & & & 0,38 \\
\hline AO & & & & & & & & & & & \\
\hline
\end{tabular}

*Valores superiores a |0,7| são considerados altamente correlacionados (em negrito).

Através da MANOVA foi possível identificar diferença significativa entre as áreas $(F=10,91 ; p<$ 0,001), e a comparação dos caracteres anatômicos revelaram variações consideráveis entre os indivíduos analisados. Na maioria das variáveis morfoanatômicas os indivíduos da Reserva FA apresentaram medidas significativamente maiores que as outras duas áreas (Tabela 3). Entretanto, o índice de plasticidade se mostrou baixo (média 0,27 ), sendo a Dest, a variável com maior valor de plasticidade.

A análise da anatomia foliar mostrou que, em vista frontal, Eumachia kappleri possui células epidérmicas de paredes retas, (Fig. 2A); é hipoestomática, sendo os estômatos do tipo paracítico no mesmo nível das demais células epidérmicas adjacentes e possui células-guarda de formato reniforme (Fig. 2B). Em secções transversais, a epiderme é uniestratificada com paredes e cutícula delgadas (Figs. 2C-E). A espessura de ambas as faces da epiderme foi maior para indivíduos de Reserva FA (Fig. 2C).

O mesofilo, com aspecto dorsiventral, possui de uma a duas camadas de parênquima paliçádico e em média cinco camadas de parênquima lacunoso com espaços intercelulares inconspícuos (Figs. 2A-C). Foi clara a diferença na espessura dos parênquimas do mesofilo da espécie entre as áreas (Figs. 2C-E). Desta maneira, também foi observado que a maior espessura do mesofilo para a espécie ocorrente foi na Reserva FA (Fig. 2C). Em relação ao feixe vascular, na nervura central a espécie possuiu feixe do tipo colateral em formato 
côncavo-convexo com face abaxial proeminente (Figs. 2F-H). Nota-se a presença de tricomas tectores nos indivíduos ocorrentes no Parque C-E (Fig. 2H).

Tabela 3: Valores médios dos atributos anatômicos foliares mensurados em Eumachia kappleri ocorrente em Floresta Ombrófila Densa Submontana e Floresta Estacional Semidecidual na Amazônia mato-grossense, Brasil.

\begin{tabular}{|l|l|l|l|l|}
\hline VARIÁVEL & Reserva FA & RPPN & Parque C_E & IPF \\
\hline EAD & $37,42 \pm 4,29 \mathrm{a}$ & $32,51 \pm 3,60 \mathrm{~b}$ & $29,30 \pm 7,70 \mathrm{~b}$ & 0,24 \\
\hline EAB & $38,36 \pm 7,62 \mathrm{a}$ & $31,33 \pm 3,19 \mathrm{~b}$ & $28,99 \pm 5,56 \mathrm{~b}$ & 0,27 \\
\hline EPA & $40,71 \pm 4,67 \mathrm{a}$ & $36,66 \pm 4,34 \mathrm{~b}$ & $29,52 \pm 7,60 \mathrm{c}$ & 0,29 \\
\hline ELA & $148,49 \pm 31,48 \mathrm{a}$ & $90,48 \pm 16,85 \mathrm{~b}$ & $92,71 \pm 22,17 \mathrm{~b}$ & 0,38 \\
\hline EME $^{\text {ns }}$ & $189,21 \pm 22,17$ & $127,15 \pm 22,17$ & $122,24 \pm 44,35$ & 0,32 \\
\hline EFNC $^{\text {DEST }}{ }^{\mathrm{n}}$ & $546,68 \pm 141,38 \mathrm{a}$ & $440,63 \pm 102,83 \mathrm{~b}$ & $414,79 \pm 59,05 \mathrm{~b}$ & 0,20 \\
\hline IE & $87,66 \pm 19,79$ & $86,74 \pm 15,91$ & $92,85 \pm 20,38$ & 0,40 \\
\hline MEE & $11,50 \pm 2,16 \mathrm{a}$ & $10,69 \pm 1,90 \mathrm{~b}$ & $9,99 \pm 2,32$ & 0,32 \\
\hline MPE & $29,60 \pm 3,51 \mathrm{a}$ & $30,80 \pm 3,94 \mathrm{a}$ & $29,98 \pm 4,08 \mathrm{a}$ & 0,18 \\
\hline AO & $18,59 \pm 2,42 \mathrm{a}$ & $18,72 \pm 2,14 \mathrm{a}$ & $16,64 \pm 2,15 \mathrm{~b}$ & 0,10 \\
\hline & $4,72 \pm 1,06 \mathrm{a}$ & $3,64 \pm 0,94 \mathrm{~b}$ & $4,314 \pm 0,81 \mathrm{a}$ & 0,28 \\
\hline
\end{tabular}

*Definição das siglas na tabela 1. Letras diferentes na linha indicam diferença significativa entre ambientes $(p<0,01)$ pelo teste de Tukey. ns: Não significativo, IPF: índice de plasticidade fenotípica, médias e desvio-padrão ( \pm ).

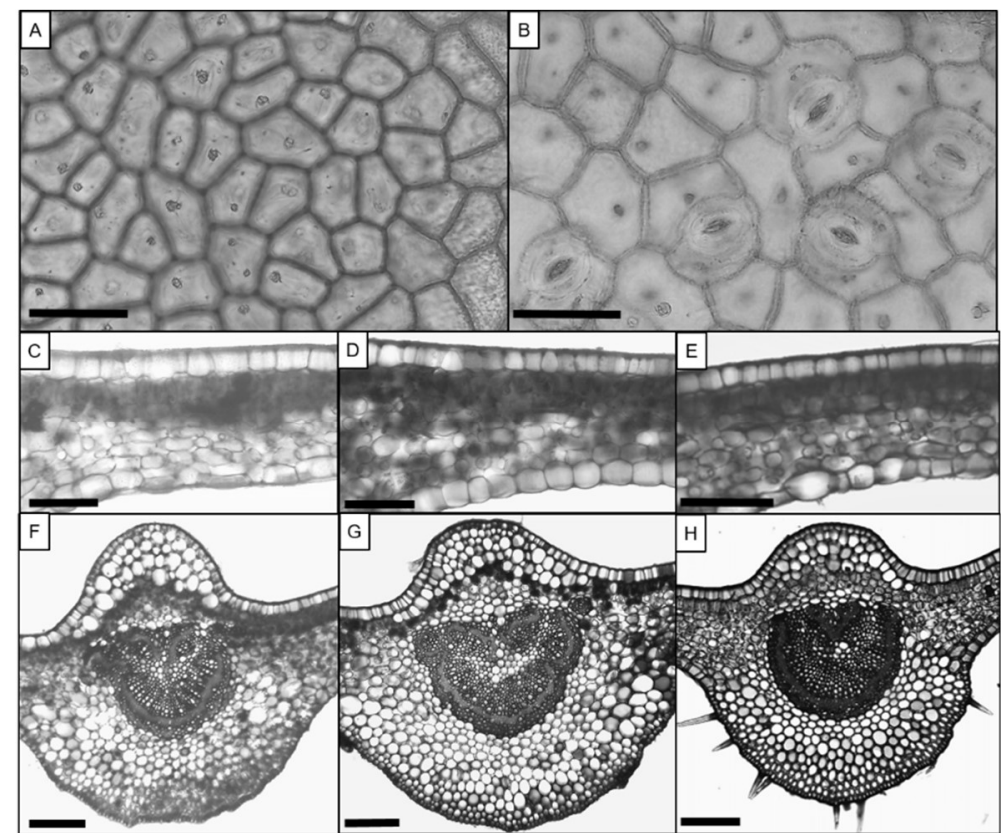

Figura 2: Secções anatômicas da lâmina foliar de Eumachia kappleri. Vista paradérmica da epiderme adaxial (A) e abaxial (B). Vista transversal do mesofilo ocorrente na Reserva FA (C); RPPN Mirante da Serra (D) e Parque C_E (E). Vista transversal da nervura central ocorrente em Reserva FA (F); RPPN Mirante da Serra (G) e Parque C-E (H). Barras: A, B e C: $50 \mu \mathrm{m} ;$ C, D e E: $100 \mu \mathrm{m} ; \mathrm{F}, \mathrm{G}$ e H: $200 \mu \mathrm{m}$.

\section{DISCUSSÃO}

Espécies de Rubiaceae possuem grande importância para estudos relacionados a ecologia, conservação e preservação em regiões tropicais (DELPRETE et al., 2012). Normalmente encontradas no subbosque de florestas úmidas neotropicais, as espécies da família possuem grande importância como fonte alimentar para a fauna, em especial para aves (BREMER et al., 1992)

A espécie estudada apresenta características anatômicas que são consideradas comuns à família, como folhas hipoestomática, estômatos paracíticos, mesofilo dorsiventral e feixes vasculares colaterais (METCALFE et al., 1979). Porém, quando comparadas em ambientes distintos, diferem em alguns aspectos; 
principalmente quanto a espessura das epidermes abaxial e adaxial, espessura dos parênquimas paliçádico e lacunoso da asa foliar; espessura do feixe da nervura central e também quanto à presença de tricomas.

Essas alterações podem estar associadas à fatores abióticos de cada ecossistema, que se diferem principalmente quanto a quantidade de luz, umidade e proximidade de cursos de água (ESAU, 2000; RABELO et al., 2013; SOMAVILLA et al., 2014). É importante ressaltar que a plasticidade observada pode ser reduzida pela limitação de recursos ou pelo estresse ambiental (VALLADARES et al., 2007).

Em plantas terrestres, a epiderme foliar é essencial para o controle do crescimento, proteção e interações ambientais (MALIVERT et al., 2018). A epiderme mais espessa em ambas as faces para os indivíduos de sub-bosque da Reserva FA pode ser justificada pelo fato de que, nessa área, os indivíduos encontram-se na borda da trilha, recebendo maior luminosidade.

O espessamento da epiderme pode ocorrer pela influência da intensidade luminosa (RAVEN et al., 2001), sendo uma resposta comum em plantas de ambientes fragmentados; está ligada ainda a incidência de ventos, que aumentam a possibilidade de perda de água por evapotranspiração (SOMAVILLA et al., 2014).

Das áreas estudadas, Reserva FA apresenta outra característica que a torna única: o fato de que os indivíduos analisados estão mais distantes do curso d'água; parâmetros anatômicos foliares como aumento da espessura da epiderme adaxial podem estar diretamente relacionados ao estresse hídrico (BOUGHALLEB et al., 2011). De fato, há estudos que evidenciam que as únicas características que variaram significativamente em resposta ao déficit hídrico são a espessura da epiderme adaxial e o volume das cavidades no parênquima lacunoso; o segundo carácter, ao contrário da expectativa, pode aumentar em resposta à seca imposta (BINKS et al., 2016).

Parênquima paliçádico e lacunoso bem desenvolvidos em relação aos tecidos protetores como a epiderme, é comumente encontrado em espécies de plantas submetidas a altas intensidades de luz (SOMAVILLA et al., 2014). A maior espessura do parênquima paliçadico não está relacionada a capacidade fotossintética máxima (BINKS et al., 2016), uma vez que as plantas investem em fotoassimilados e na expansão dos tecidos fotossintéticos, maximizando a interceptação de luz e, consequentemente, o crescimento (DALE, 1988; BOUGHALLEB et al., 2011). Entretanto, a espessura desse tecido pode aumentar em resposta ao déficit de água, influenciando o uso da água dentro da folha (BOUGHALLEB et al., 2011), assim como evidenciado pelos indivíduos da Reserva FA, estando estes, mais distantes de cursos d'água.

Os parâmetros da fotossíntese estão positivamente correlacionados com a espessura do mesofilo como um todo (BINKS et al., 2016). O incremento da espessura do mesofilo através do investimento em parênquima lacunoso pode atuar como estratégia para otimizar processos fotossintéticos (TIAN et al., 2016; SAFIALLAH, 2017), sendo útil para plantas que enfrentam a sazonalidade hídrica. Um mesofilo espesso pode influenciar aumentando potencialmente a contundência dentro da folha e reduzindo a necessidde de baixo potêncial osmótico, necessário para manter o tugor com baixa disponibilidade de água (BINKS et al., 2016). Já o aumento das dimensões do feixe vascular é fundamental para eficiência do transporte de nutrientes e água (TAIZ et al., 2008). Fatores relacionados ao ambiente afetam as dimensões e o arranjo dos elementos vasculares, uma tentativa de garantir aumento na segurança do transporte (ALVES et al., 2000). 


\section{CONCLUSÕES}

A hipótese de que existe influência do espaço, não se aplica às áreas estudadas de acordo com os resultados obtidos, porém estes inferem que as diferenças anatômicas entre as populações amostradas são decorrentes de condições ambientais. Pode-se afirmar também, que algumas das variações anatômicas entre as amostras analisadas favorecem a aclimatação da espécie às clareiras florestais e que E. kappleri é bem ajustada a ambientes abertos, apresentando diferenças relevantes dentro das populações. Concluise ainda, que os indivíduos estudados da população da Reserva FA sofreram ajustes estruturais, como a espessura de epiderme adaxial e abaxial e espessura de parênquima paliçádico e lacunoso, provavelmente devido às diferentes condições de luminosidade e disponibilidade de água.

\section{REFERÊNCIAS}

AGUIAR, T. V.; SANT'ANNA-SANTOS, B. F.; AZEVEDO, A. A.; FERREIRA, R. S.. Anati quanti: software de análises quantitativas para estudos em anatomia vegetal. Planta Daninha, v.25, n.4, p.649-659, 2007.

ALVES, E. S.; ANGYALOSSY-ALFONSO, V.. Ecological trends in the Wood of some Brazilian species 1: growth rings and vessels. IAWA Jornal, v.21, p.03-30, 2000.

ANDERSSON, L. Margaritopsis (Rubiaceae, Psychotrieae) is a pantropical genus. Systematics and Geography of Plants, v.71, n.1, p.73-85, 2001.

ANDERSSON, L.; ROVA, J. H.. Therps16 intron and the phylogeny of the Rubioideae (Rubiaceae). Plant Systematics and Evolution, v.214, n.1-4, p.161-186, 1999.

BINKS, O.; MEIR, P.; ROWLAND, L.; COSTA, A. C. L.; VASCONCELOS, S. S.; OLIVEIRA, A. A. R.; MENCUCCINI, M.. Limited acclimation in leaf anatomy to experimental drought in tropical rainforest trees. Tree physiology, v.36, n.12, p.1550-1561, 2016.

BOUGHALLEB, F.; HAJLAOUI, H.. Physiological and anatomical changes induced by drought in two olive cultivars (cv Zalmati and Chemlali). Acta Physiologiae Plantarum, v.33, n.1, p.53-65, 2011.

BREMER, B.; ERIKSSON, O.. Evolution of fruit characters and dispersal modes in the tropical family Rubiaceae. Biological Journal of the Linnean Society, v.47, p.79-95, 1992.

CABRAL, R. D. C.; MELO JUNIOR, J. C. F. D.; MATILDE-SILVA, M.. Foliar morphoanatomical plasticity of Smilax campestris (Smilacaceae) in an environmental gradient of restinga, Santa Catarina State, Brazil. Hoehnea, v.45, n.2, p.173-183, 2018.

CAMPBELL, G.; MIELKE, M. S.; RABELO, G. R.; CUNHA, M. Key anatomical attributes for occurrence of Psychotria schlechtendaliana (Müll. Arg.) Müll. Arg.(Rubiaceae) in different successional stages of a tropical moist forest. Flora, v.246, p.33-41, 2018.

CAO, K. F.. Leaf anatomy and chlorophyll content of 12 woody species in contrasting light conditions in a Bornean heath forest. Canadian Journal of Botany, v.78, n.10, p.1245-1253, 2000.

CATONI, R.; GRANATA, M. U.; SARTORI, F.; VARONE, L.; GRATANI, L.. Corylus avellana responsiveness to light variations: morphological, anatomical, and physiological leaf trait plasticity. Photosynthetica, v.53, n.1, p.35-46, 2015.

DALE, J. E.. The control of leaf expansion. Annual Review Plant Physiology, v.39, p.267-295, 1988.

DARDENGO, J. D. F. E.; ROSSI, A. A. B.; SILVA, I. V. D.; PESSOA, M. J. G.; SILVA, C. J. D.. Analysis of the luminosity on the anatomical aspects of Theobroma speciosum willd. Ex spreng. (malvaceae) leaves. Ciência Florestal, v.27, n.3, p.843-851, 2017.

DELPRETE, P. G.; JARDIM, J. G.. Systematics, taxonomy and floristics of Brazilian Rubiaceae: an overview about the current status and future challenges. Rodriguésia, v.63, n.1, p.101-128, 2012.

DUBREUIL, V.; DEBORTOLI, N.; FUNATSU, B.; NÉDÉLEC, V.; DURIEUX, L.. Impact of land-cover change in the Southern Amazonia climate: a case study for the region of Alta Floresta, Mato Grosso, Brazil. Environmental monitoring and assessment, v.184, n.2, p.877-891, 2012.

EMBRAPA. Empresa Brasileira de Pesquisa Agropecuária Embrapa Florestas. 2018.

ESAU, K.. Anatomia das plantas com sementes. São Paulo: Edgard Blücher, 2000.

FOX, J.; WEISBERG, S.; ADLER, D.; BATES, D.; BAUD-BOVY, G.; ELLISON, S.; HEIBERGER, R.. Package 'car'. Vienna: $R$ Foundation for Statistical Computing, 2012.

FUNDAÇÃO CRISTALINO. Incalculável valor para a Biodiversidade. 2018.

GAO, F.; CALATAYUD, V.; GARCÍA-BREIJO, F.; REIGARMIÑANA, J.; FENG, Z.. Effects of elevated ozone on physiological, anatomical and ultrastructural characteristics of four common urban tree species in China. Ecological Indicators, v.67, p.367-379, 2016. 
GAVILANES, M. L.; CASTRO, E. M.; FERREIRA PIRES, M.; PEREIRA, F. J.; PEREIRA, M. P.. Micromorfometria foliar de Palicourea rigida Kunth. Rubiaceae) em ambiente de Cerrado e Campo Rupestre. CERNE, v.22, n.2, p.163-170, 2016.

HAMMER, O.; HARPER, A. T. D.; RYAN, P. D.. Past: Paleontological Statistics Software Package for Education and Data Analysis Palaeontologia Electronica. 2001.

HØJSGAARD, S.; HALEKOH, U.; ROBISON-COX, J.; WRIGHT, K.; LEIDI, A. A.. doBy: Groupwise statistics, LSmeans, linear contrasts, utilities. R package version, v.4, p.5-13, 2014.

IBGE. Manual técnico da vegetação brasileira: sistema fitogeográfico, inventário das formações florestais e campestres, técnicas e manejo de coleções botânicas, procedimentos para mapeamentos. Rio de Janeiro, 2012.

JOHANSEN, D. A.. Plant microtechnique. London: McGrawHill Book Company Inc, 1940.

KRAUS, J. E.; ARDUIN, M.. Manual básico de métodos em morfologia vegetal. Rio de Janeiro: Edur, 1997.

MALIVERT, A.; HAMANT, O.; INGRAM, G.. A contribuição da mecanossensibilização para a especificação do destino das células epidérmicas. Opinião atual em genética e desenvolvimento, v.51, p.52-58, 2018.

MELO JÚNIOR, J. C. F. D.; SILVA, M. M. D.; AMORIM, M. W.; TUSSOLINI, E. G. R.. Structural adaptations of seven shade shrubs in species of Tropical Rain Forest. Hoehnea, v.44, n.2, p.193-201, 2017.

METCALFE, C. R.; CHALK, L.. Anatomy of Dicotyledons. Oxford: Oxford University Press, 1979.

MORAES, T. M. D. S.; RABELO, G. R.; ALEXANDRINO, C. R.; SILVA NETO, S. J. D.; CUNHA, M.. Comparative leaf anatomy and micromorphology of Psychotria species (Rubiaceae) from the Atlantic Rainforest. Acta Botanica Brasilica, v.25, n.1, p.178-190, 2011.

NEPOKROEFF, M.; BREMER, B.; SYTSMA, K. J.. Reorganization of the genus Psychotria and tribe Psychotrieae (Rubiaceae) inferred from ITS and rbcL sequence data. Systematic Botany, v.24, n.1, p.5-27, 1999.

R CORE TEAM. R: A language and environment for statistical computing. R Foundation for Statistical Computing, Vienna, Austria. 2017.

RABELO, G. R.; VITÓRIA, Â. P.; SILVA, M. V.; CRUZ, R. A.; PINHO, E. I.; RIBEIRO, D. R.; CUNHA, M.. Structural and ecophysiological adaptations to forest gaps. Trees, v.27, n.1, p.259-272, 2013.
RAVEN, P. H.; EVERT, R. F.; EICHHORN, S. E.. Biologia

Vegetal. Rio de Janeiro: Guanabara, Koogan S.A., 2001.

RÔÇAS, G.; SCARANO, F. R.; BARROS, C. F.. Leaf anatomical variation in Alchornea triplinervia (Spreng) Müll. Arg.

(Euphorbiaceae) under distinct light and soil water regimes. Botanical Journal of the Linnean Society, v.136, n.2, p.231238, 2001.

SILVA, M. M.; MELO JÚNIOR, J. C. F.. Plasticidade da folha e lenho de cinco espécies lenhosas em duas áreas de restinga no Sul do Brasil. Iheringia. Série Botânica, v.72, n.2, p.173180, 2017.

SAFIALLAH, S.; HAMDI, S. M. M.; GRIGORE, M. N.; JALILI, S.. Micromorphology and leaf ecological anatomy of Bassia halophyte species (Amaranthaceae) from Iran. Acta Biologica Szegediensis, v.61, n.1, p.85-93, 2017.

SASAKI, D.; ZAPPI, D.; MILLIKEN, W.; HENICKA, G. D. S.; PIVA, J. H.. Vegetação e plantas do Cristalino-um manual. Alta Floresta, Royal Botanic Gardens. 2010.

SEMA. Plano de Manejo do Parque Estadual Cristalino. Portaria n.031. Diário Oficial do Estado de Mato Grosso, 2010.

SOMAVILLA, N. S.; KOLB, R. M.; ROSSATTO, D. R.. Leaf anatomical traits corroborate the leaf economic spectrum: a case study with deciduous forest tree species. Brazilian Journal of Botany, v.37, n.1, p.69-82, 2014.

TAIZ, L.; ZEIGER, E.. Fisiologia vegetal. 4 ed. Porto Alegre: ARTMED, 2008.

TAYLOR, C. M.; RAZAFIMANDIMBISON, S. G.; BARRABÉ, L.; JARDIM, J. G.; BARBOSA, M. R. V.. Eumachia expanded, a pantropical genus distinct from Psychotria (Rubiaceae, Palicoureeae). Candollea, v.72, n.2, p.289-318, 2017.

TIAN, M.; YU, G.; HE, N.; HOU, J.. Leaf morphological and anatomical traits from tropical to temperate coniferous forests: Mechanisms and influencing factors. Scientific reports, v.6, p.19703, 2016

VALLADARES, F.; GIANOLI, E. R.; GÓMEZ, J. M.. Ecological limits to plant phenotypic plasticity. New Phytologist, v.176, n.4, p.749-763, 2007.

WICKHAM, H.; FRANCOIS, R.; HENRY, L.; MÜLLER, K.. Dplyr: A Grammar of Data Manipulation. R package version 0.7.6. 2018.

ZINI, A. S.; MARTINS, S.; TODERKE, M. L.; TEMPONI, L. G. Anatomia foliar de Rubiaceae ocorrentes em fragmento florestal urbano de Mata Atlântica, PR, Brasil. Hoehnea, v.43, p.173-182, 2016.

A CBPC - Companhia Brasileira de Produção Científica (CNPJ: 11.221.422/0001-03) detém os direitos materiais desta publicação. Os direitos referem-se à publicação do trabalho em qualquer parte do mundo, incluindo os direitos às revacões, expansões e disseminoc̃os da contribuiç̃o, bem como outros direitos subsidiários. Todos os trabalhos publicados eletronicamente poderão posteriormente ser publicados em coletâneas impressas sob coordenação da Sustenere Publishing da Companhia Brasileira de Produção Científica e seus parceiros autorizados. Os (as) autores (as) posteriormente ser publicados em coletâneas impressas sob coordenação da Sustenere Publishing, da Companhia Brasileira de Produção Cientifica e seus pa 